\title{
Antitumor Activity of Hot-Water Extract from Delipidated BCG
}

\author{
Hirosi Sato, Atsunobu Yokosawa, Hideo Arai, Hiromi \\ Nagai, Nobuko Kumano, Masakichi Motomtya and \\ KIYOSHI Konno \\ The Research Institute for Tuberculosis and Cancer, Tohoku \\ University, Sendai 980
}

\begin{abstract}
Sato, H., Yokosawa, A., Arai, H., NagaI, H., Kumano, N., Motomiya, M. and Konvo, K. Antitumor Activity of Hot-Water Extract from Delipidated BCG. Tohoku J. exp. Med., 1978, 125 (3), 247-252 — The antitumor activity of hotwater extract of delipidated BCG was investigated in mice inoculated with Sarcoma-180 cells and Ehrlich carcinoma cells, respectively. The hot-water extract was found to be effective when administered after and ineffective when administered before the inoculation of tumor cells. When this extract was given with anticancer drugs, such as Mitomycin $\mathrm{C}$ and cyclophosphamide, a combined effect was obtained in the treatment of Sarcoma-180 and of Ehrlich carcinoma. water-soluble adjuvant; $\mathrm{BCG}$
\end{abstract}

The mycobacterial preparations such as whole bacilli of $B C G$, cell wall skeleton (Meyer et al. 1974), methanol extraction residue (Shlomo et al. 1973), and cord factor (trehalose dimycolate) (Bekierkunst 1976), which are all adjuvant-active, have been employed in the treatment of neoplasms in recent years. Also the adjuvant activity of these preparations is known to be essential for their antitumor activity.

Since 1972, several water-soluble substances from delipidated mycobacteria (Adam et al. 1973; Migliore-Samour and Jollès 1972, 1973) and cell walls (Adam et al. 1972) have been reported to possess adjuvant activity.

The hot-water extract obtained from defatted Mycobacterium bovis strain BCG was found to have adjuvant activity and was designated as HSA (Hot-water Soluble Adjuvant) in our previous paper (Sato et al. 1976).

In the present study the antitumor effect of the HSA on the intraperitoneal inoculum of Sarcoma-180 and Ehrlich carcinoma in mice was investigated.

\section{Materials and Methods}

Throughout the experiments, 4-week-old ddI mice of both sexes weighing $22-24 \mathrm{~g}$ were employed. Sarcoma-180 and Ehrlich carcinoma in ascitic form have been maintained in daI mice by weekly inoculations. In each experiment the suspension of the tumor cells was diluted with saline immediately before use and the desired number of the cells were inoculated intraperitoneally. The viability of cells was examined by the dye exclusion test.

Received for publication, December 7, 1977. 
The hot-water extract was prepared as previously reported (Sato et al. 1976) and was taken up in $0.25 \mathrm{ml}$ of saline and injected intraperitoneally.

Antitumor effect on Sarcoma-180

Experiment 1. Mice were divided into 3 equal groups of 20 mice. The first group received intraperitoneal injections of the HSA for 7 consecutive days before inoculation of $3 \times 10^{\circ}$ tumor cells. The second group was treated with intraperitoneal injections of HSA for 7 consecutive days after inoculation of the tumor cells. The third group was used as a control group. The number of the survivors was recorded in the above three groups (Fig. 1).

Experiment II. Eighty mice were divided into 4 equal groups of 20 mice. Three of the four gronps (control, Group $I$ and Group 2 in Fig. 2) were treated as in Experiment I. The fourth group (Group 3 in Fig. 2) received an injection of HSA for 5 consecutive days before and after inoculation of $3 \times 10^{6}$ tumor cells.

Experiment III. Four groups of mice each consisting of 20 mice were inoculated with $3 \times 10^{6}$ tumor cells. The first group received an injection of Mitomycin $\mathrm{C}(1 \mathrm{mg} / \mathrm{kg})$ in saline at $1,3,5$ and 7 days after inoculation of the tumor cells. The second group received 7 consecutive injections of HSA from 1 day after inoculation of the tumor cells. The third group received Mitomycin C and HSA in the same manner as in the first and second groups. The fourth group served as a control.

Antitumor effect on Ehrlich carcinoma

Experiment I. Sixty mice were divided into 3 equal groups of 20 mice. Group A received 7 intraperitoneal injections of $0.25 \mathrm{mg}$ of HSA every day before inoculation of the tumor cells $\left(1.2 \times 10^{7}\right)$. Group B received the same dose of HSA for 7 consecutive days after inoculation of the tumor cells. The third group received saline alone and was used as a control. The mean survival time in terms of days was compared among the above three groups (Table 1).

Experiment II. Eighty mice were divided into 4 groups of 20 mice each. In this experiment the size of inoculum was reduced to $1.0 \times 10^{5}$ cells. Group 1 received HSA for 7 consecutive days prior to and Group 2 after the inoculation of tumor cells. Group 3 received HSA for 5 conseuctive days before and after inoculation of the tumor cells. The fourth control group received the injections of saline alone.

Experiment III. One hundred mice received an inoculation of $2 \times 10^{6}$ tumor cells. 2 and $4 \mathrm{mg}$ of cyclophosphamide in saline were given to two groups of mice at 2 days after inoculation of the tumor cells. The other two groups were injected with cyclophosphamide in the same manner as in the former two groups and were treated with HSA for 7 consecutive days from 2 days after inoculation of the tumor cells. The numbers of survivors were compared among four experimental groups and the control (Table 2).

\section{Results}

\section{Antitumor effect on Sarcoma-180}

Fig. 1 shows the results of the experiment in which HSA was injected for 7 consecutive days each before or after inoculation of Sarcoma-180 cells. The survival time of the mice received HSA before inoculation of the tumor cells was prolonged only slightly. On the other hand an increase of survival time was evident in the mice treated with HSA after inoculation of the sarcoma cells.

The results of another experiment in which HSA alone was administered are summarized in Fig. 2. In this experiment survival time was not prolonged in the mice that had received HSA before inoculation of the tumor cells. In the group which received HSA for 5 consecutive days before and after inoculation of the tumor cells only a very slight effect was observed. 


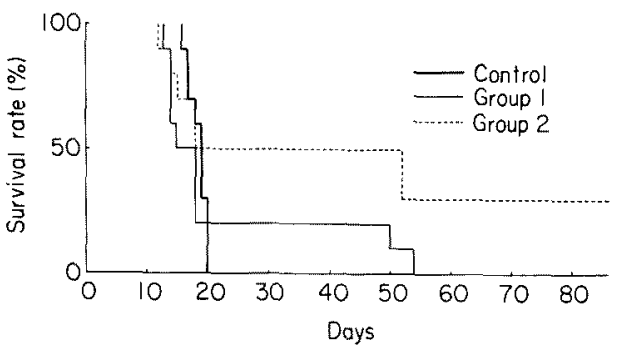

Fig. 1. Antitumor activity of HSA on Sarcoma-180.

Group 1, injected intraperitoneally with $0.25 \mathrm{mg}$ of HSA for 7 consecutive days before intraperitoneal inoculation of $3 \times 10^{6}$ tumor cells; Group 2, injected intraperitoneally with $0.25 \mathrm{mg}$ of HSA for 7 consecutive days after intraperitoneal inoculation of $3 \times$ $10^{6}$ tumor cells.

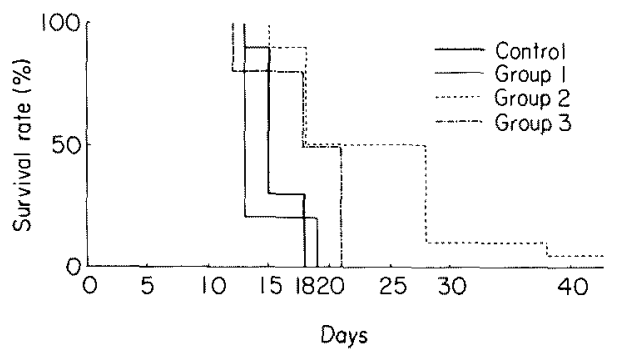

Fig. 2. Antitumor activity of HSA on Sarcoma-180.

Group 1, received $0.3 \mathrm{mg}$ of HSA intraperitoneally for 7 consecutive days before intraperitoneal inoculation of $3 \times 10^{6}$ tumor cells; Group 2, received $0.3 \mathrm{mg}$ of HSA intraperitoneally for 7 consecutive days after intraperitoneal inoculation of $3 \times 10^{6}$ tumor cells; Group 3, received $0.3 \mathrm{mg}$ of HSA intraperitoneally for 5 consecutive days before and after intraperitoneal inoculation of $3 \times 10^{6}$ tumor cells, respectively.

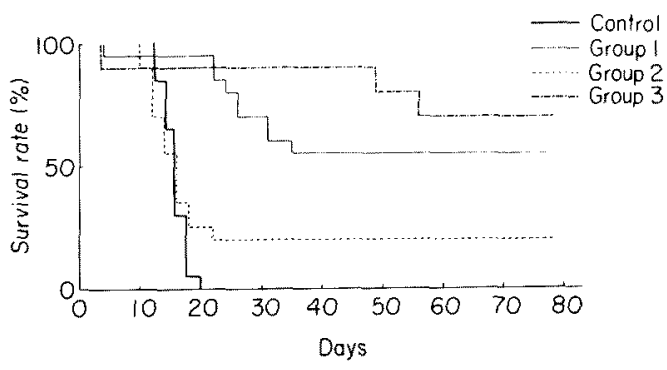

Fig. 3. Effect of combined regimen of Mitomycin C and HSA on Sarcoma-180.

Group 1, given $0.02 \mathrm{mg}$ of Mitomycin C intraperitoneally at $1,3,5$ and 7 days after intraperitoneal inoculation of $3 \times 10^{6}$ tumor cells; Group 2, given $0.25 \mathrm{mg}$ of HSA intraperitoneally for 7 consecutive days after intraperitoneal inoculation of $3 \times 10^{6}$ tumor cells; Group 3, given both Mitomycin C and HSA in the same manner as in Group 1 and Group 2. 
The combined effect of HSA plus Mitomycin C is demonstrated in Fig. 3. In experiment III the survival time became longer in the mice treated with Mitomycin $\mathrm{C}$ alone. In addition it was found that in the group treated with both HSA and Mitomycin $\mathrm{C}$ the survival time became markedly prolonged.

TABLE 1. Antitumor activity of HSA on Ehrlich carcinoma

\begin{tabular}{ccc} 
Group & Number of mice & $\begin{array}{c}\text { Survival days } \\
\text { (mean } \pm \text { s.D.) }\end{array}$ \\
\hline Control & 20 & $16.0 \pm 3.2$ \\
A & 20 & $14.3 \pm 1.6$ \\
B & 20 & $11.2 \pm 3.0$ \\
\hline
\end{tabular}

Group A: Injected intraperitoneally with $0.25 \mathrm{mg}$ of HSA for 7 consecutive days before intraperitonal inoculation of $1.2 \times 10^{7}$ tumor cells.

Group B: Injected intraperitoneally with $0.25 \mathrm{mg}$ of HSA for 7 consecutive days after intraperitoneal inoculation of $1.2 \times 10^{7}$ tumor cells.

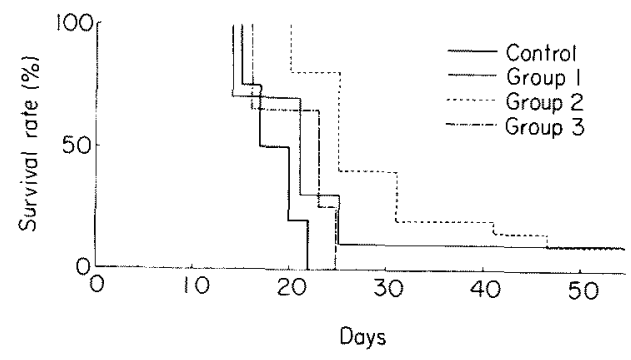

Fig. 4. Antitumor activity of HSA on Ehrlich carcinoma

Group 1, injected intraperitoneally with $0.3 \mathrm{mg}$ of HSA for 7 consecutive days before intraperitoneal inoculation of $1.0 \times 10^{5}$ tumor cells; Group 2, injected intraperitoneally with $0.3 \mathrm{mg}$ of HSA for 7 consecutive days after intraperitoneal inoculation of $1.0 \times 10^{5}$ tumor cells; Group 3 , injected intraperitoneally with $0.3 \mathrm{mg}$ of HSA for 5 consecutive days before and after intraperitoneal inoculation of $1.0 \times 10^{5}$ tumor cells respectively.

\section{Antitumor effect on Ehrlich carcinoma}

Table 1 summarizes the results of the effect of HSA on Ehrlich carcinoma. In this experiment, $1.2 \times 10^{7}$ cells were inoculated. No antitumor effect was obtained either in the group which had received HSA before inoculation or in that which was treated with HSA after inoculation of the tumor cells. When the size of inoculum was reduced to $1.0 \times 10^{5}$, as in Fig. 4 the survival time became longer in the mice which received HSA after inoculation of the tumor cells. However no effect was observed in the group treated with HSA before inoculation of the tumor cells. 
Table 2 demonstrates the results of the effect of cyclophosphamide in combination with HSA. The survival time of the mice which had received cyclophosphamide alone was longer, when compared with those of the control group. In the groups which received HSA in addition to cyclophosphamide, the survival time was more markedly prolonged than in the cyclophosphamide-alone groups.

TABLE 2. Effect of combined regimen of cyclophosphamide and HSA on Ehrlich carcinoma

\begin{tabular}{ccccc}
\hline Group & Number of mice & \multicolumn{1}{c}{ Treatment } & $\begin{array}{c}\text { Survival days } \\
\text { (mean } \pm \text { s.D.) }\end{array}$ \\
\cline { 1 - 1 } 1 & 20 & None & $16.6 \pm 3.5$ \\
2 & 20 & CY $100 \mathrm{mg} / \mathrm{kg}$ & $22.4 \pm 3.1$ \\
3 & 20 & CY $200 \mathrm{mg} / \mathrm{kg}$ & $23.4 \pm 5.1$ \\
4 & 20 & CY $100 \mathrm{mg} / \mathrm{kg}+\mathrm{HSA}$ & $25.9 \pm 4.2$ \\
5 & 20 & CY $200 \mathrm{mg} / \mathrm{kg}+\mathrm{HSA}$ & $27.3 \pm 5.2$ \\
\hline
\end{tabular}

CY, cyclophosphamide.

Groups 2 and 3 received $2 \mathrm{mg}$ and $4 \mathrm{mg}$, respectively, of cyclophosphamide by intraperitoneal injection 2 days after intraperitoneal inoculation of $2 \times 10^{6}$ tumor cells.

Groups 4 and 5 received $0.3 \mathrm{mg}$ of HSA intraperitoneally in addition to cyclophosphamide given in the same manner as in Groups 2 and 3.

\section{Discussion}

It is well known that the adjuvant activity is closely related to antitumor effects. Hiu (1972) reported a remarkable antitumor effect of a water-soluble adjuvant on L1210 leukemic tumor cells. According to the reports of Mathé et al. (1973, 1975), mycobacterial water-soluble substance of Hiu showed only a very slight antitumor activity when administered by a single injection. Juy et al. (1974) demonstrated an in vitro antitumor effect of the peritoneal macrophages when stimulated by the water-soluble adjuvant which was prepared as reported by Adam et al. However there have been only a few reports concerning the antitumor activity in vivo of the water-soluble adjuvants from mycobacterial preparations. When HSA was given only once in this experiment, no effect was found on Sarcoma-180. Thus it is likely that the water-soluble adjuvant exerts antitumor activity only after repeated injections. Presumably low yield of watersoluble adjuvants has made it diffcult to prepare a large quantity of the material for repeated injections. For similar reasons the dose and duration for satisfactory treatment with HSA could not be established in this experiment. There have been several reports stating that immunotherapy is less effective when a large number of tumor cells are inoculated (Bast et al. 1974). In the present study HSA was effective when $1 \times 10^{5}$ cells of Ehrlich carcinoma were inoculated, but the effect was very slight when $1.2 \times 10^{7}$ cells were inoculated. It is not known whether the combined effect of HSA plus antitumor drugs such as Mitomycin $\mathrm{C}$ and cyclophosphamide is due to a decrease in the number of tumor cells by the effect of 
cytostatic agents or to the recovery of the function of reticuloendothelial systems which had been depressed by antitumor drugs.

The reason why the injection of HSA prior to the inoculation of tumor cells was ineffective remains to be clarified. Experiments are now being carried out along this line.

\section{References}

1) Adam, A., Ciorbaru, R., Petit, J.F. \& Lederer, E. (1972) Isolation and properties of a macromolecular, water-soluble immunoadjuvant fraction from cell wall of $\mathrm{My}$ cobacterium smegmatis. Proc. nat. Acad. Sci. (Wash.), 69, 851-854.

2) Adam, A., Ciorbaru, R., Petit, J.F., Lederer, E., Chedid, L., Lamensans, A., Parant, F., Parant, M., Rosselet, J.F. \& Berger, F.M. (1973) Preparation and biological properties of water-soluble adjuvant fractions from delipidated cells of Mycobacterium smegmatis and Nocardia opaca. Infect. Immun., 7, 855-861.

3) Bast, R.C., Zbar, B., Borsos, T. \& Rapp, H.J. (1974) BCG and cancer (First of two parts). New Engl. J. Med., 290, 1413-1420.

4) Bekierkunst, A. (1976) Immunotherapy of cancer with nonliving mycobacteria and cord factor (trehalose-6,6 $6^{\prime}$-dimycolate) in aqueous medium. $J$. nat. Cancer Inst., 57, 963-964.

5) Hiu, I.J. (1972) Water-soluble and lipid-free fraction from BCG with adjuvant and antitumor activity. Nature New Biol., 238, 241-242.

6) Juy, D., Bona, C. \& Chedid, L. (1974) Effet antitumoral de macrophages peritoneaux par un adjuvant hydrosoluble d'origine mycobacterienne. C.R. Acad. Sci. (Paris), 278, 2859-2862.

7) Mathé, G., Kamel, M., Dezfulian, M., Halle-Pannenko, O. \& Bourut, C. (1973) An experimental screening for "systemic adjuvants of immunity" applicable in cancer immunotherapy. Cancer Res., 33, 1987-1997.

8) Mathé, G., Halle-Pannenko, O., Florentin, I., Bruley-Rosset, M., Kamel, M., Hiu, I.J. \& Bourut, C. (1975) The second generation of EORTC-ICIG experimental screening for systemic immunity adjuvant. Europ. J. Cancer, 11, 801-807.

9) Meyer, T.J., Ribi, E.E., Azuma, I. \& Zbar, B. (1974) Biologically active components from mycobacterial cell wall. II. Suppression and regression of strain-2 guinea pig hepatoma. J. nat. Cancer Inst., 52, 103-111.

10) Migliore-Samour, D. \& Jollès, P. (1972) A hydrosoluble, adjuvant-active mycobacterial "polysaccharide-peptidoglycan'. preparation by a simple extraction technique of the bacterial cells (strain Peurois). FEBS Lett., 25, 301-304.

11) Migliore-Samour, D. \& Jollès, P. (1973) Hydrosoluble adjuvant-active mycobacterial fractions of low molecular weight. FEBS Lett., 35, 317-321.

12) Sato, H., Yokosawa, A., Arai, H., Nagai, H., Kurita, K., Kumano, N., Motomiya, M. \& Konno, K. (1976) Chemical and biological properties of hot water extract from delipidated cells of Mycobacterium bovis strain BCG. Toholu J. exp. Med., 120, 75-81.

13) Shlomo, B.E., Constanti-Sourojon, M. \& Weiss, D.W. (1973) Potentiation and modulation of the immune response of guinea pigs to poorly imnunogenic proteinhapten conjugates by pretreatment with the MER fraction of attenuated tubercle bacilli. Cellular Immunol., 7, 370-379.

14) Wainberg, M.A., [Deutsch, V. \& Weiss, D.W. (1976) Stimulation of anti-tumor immunity in guinea-pigs by methanol extraction residue of BCG. Brit. J. Cancer, 34, $500-508$. 